\title{
Review \\ Reverse genetic studies of mitochondrial DNA-based diseases using a mouse model
}

\author{
By Kazuto NAKADA*1, Akitsugu SATO*1 and Jun-Ichi HAYASHI ${ }^{* 1, \dagger}$ \\ (Communicated by Takao SEKIYA, M.J.A.)
}

\begin{abstract}
In the situation that it would not be able to produce model animals for mitochondrial diseases caused by mitochondrial DNA (mtDNA) with pathogenic mutations, we succeeded in generating mice with pathogenic deletion mutant mtDNA $(\Delta \mathrm{mtDNA})$, named "mito-mice", by direct introduction of mitochondria with $\Delta$ mtDNA into mouse zygotes. In the mito-mice, accumulation of $\triangle \mathrm{mtDNA}$ induced mitochondrial respiration defects in various tissues, resulting in mitochondrial disease phenotypes, such as low body weight, lactic acidosis, ischemia, myopathy, heart block, deafness, male infertility, and renal failure. Thus, mito-mice are the first model animal for mtDNA-based diseases, and the mice could be valuable for understanding precise pathogeneses and testing therapies of mitochondrial diseases. In the present review, we summarized reverse genetic studies using the mito-mice.
\end{abstract}

Keywords: mouse model, mitochondrial DNA, large-scale deletion mutation, mitochondrial complementation, mitochondrial diseases

\section{Introduction}

Mitochondria are one of the organelles, which can produce $90 \%$ or more of all the energy made in the body by oxidative phosphorylation. Mammalian mitochondria have multi-copies of own genome $\left(10^{3}\right.$ and $10^{4}$ copies/cell), mtDNA, that is replicated and expressed within the organellar system. ${ }^{1), 2)}$ Mammalian mtDNA encodes 13 polypeptides which are essential subunits of complexes I, III, IV, and V for oxidative phosphorylation on the inner mitochondrial membrane and 22 tRNAs and 2 rRNAs which are necessary for the translation of these 13 polypeptides. The remaining mitochondrial proteins for oxidative phosphorylation, metabolic enzymes, DNA and RNA polymerases, and ribosomal proteins are all encoded by nuclear genome. In normal individual, nearly all of the mtDNA is thought to be identical. In some cases, however, especially in mitochondrial diseases, wild-type and

*1 Graduate School of Life and Environmental Sciences, University of Tsukuba, Tsukuba, Japan.

$\dagger$ Correspondence should be addressed: J.-I. Hayashi, Graduate School of Life and Environmental Sciences, University of Tsukuba, Tsukuba, Ibaraki 305-8572, Japan

(e-mail: jih45@sakura.cc.tsukuba.ac.jp). mutant mtDNAs coexist in cells and organs.

Forward genetic studies, one of the strategies for understanding the genetic reason from phenotypes of the organism, suggested that accumulation of pathogenic mtDNAs having large-scale deletion or point mutation and the resultant mitochondrial respiratory abnormalities are associated with a wide variety of disorders, such as mitochondrial diseases, neurodegenerative diseases, and diabetes. ${ }^{3)}$ Although the pathogenicities of these mtDNA mutations were proved by co-transmission of the mutant mtDNAs and respiration defects to human mtDNA-less cells (rho-0 cells), ${ }^{4)-7)}$ there is as yet no convincing evidence to explain whether accumulation of these pathogenic mutant mtDNAs in tissues is responsible for the expressions of various clinical phenotypes.

Reverse genetic studies, one of the strategies for understanding the mechanism of phenotypic expression of organisms from genetic manipulation, could provide model mouse systems for studying exactly how pathogenic mutant mtDNA is transmitted and distributed in tissues resulting in the pathogenesis of mitochondrial diseases that show various clinical phenotypes. However, no procedures are thus far available for introduction of 
mutagenized mammalian whole mtDNA into mitochondria in living cells or even into isolated mitochondria. Therefore, generation of mouse models for mitochondrial diseases so far reported has been limited by disruption of nuclear DNA-coded factors related to mitochondrial functions. ${ }^{8)-13)}$ Adenine nucleotide translocater 1 (Ant1)-deficient mice were generated by targeted inactivation of the nuclear-coded Ant1 gene, and showed chronic ATP deficiency, myopathy, and hypertrophic cardiomyopathy. ${ }^{8)}$ Mn superoxide dismutase (MnSOD)-deficient mice were generated by inactivating mutations in the nuclear encoded SOD2 gene, and showed disease phenotypes in skeletal muscle, heart, liver, and brain. ${ }^{9)}$ Several kinds of mitochondrial transcription factor A (Tfam)-deficient mice were generated by inactivation of the nuclear Tfam gene. ${ }^{10)}$ Tissue specific disruption of the Tfam gene in cardiac muscle cells, pancreatic beta-cells, and nervous cells induced dilated cardiomyopathy, diabetes, neurodegenerative diseases, respectively. ${ }^{11)-13)}$

The effective procedure to generate mouse models carrying pathogenic mutatnt mtDNA is to introduce exogenously mitochondria carrying the mutant mtDNA by microinjection or cell fusion techniques. Using the cell fusion technique, we have succeeded in generating mice with mitochondrial dysfunction by introduction of mitochondria with somatic mutant mtDNA into mouse zygotes. ${ }^{14)}$

\section{Generation of mice carrying pathogenic deletion mutant mtDNA (mito-mice)}

In generation of mito-mice, $\Delta$ mtDNA (Fig. 1A) accumulated in mouse cells was trapped into mouse rho-0 cells ${ }^{15), 16)}$ by isolation of cybrid cells with various proportions of $\Delta \mathrm{mtDNA}$, and then respiration-deficient mitochondria were introduced into mouse zygotes using electrofusion with the enucleated cybrids (Fig. 1B).

Methodologically, there are two essential points for successful generation of mito-mice. The first point is to isolate mitochondrial donor carrying mtDNA with somatic mutations from mouse cells. Since the mutation frequency in mtDNA is about 10 times higher than that in nuclear genome, it has been known that somatic mutations occur and accumulate in mtDNA molecules with aging. ${ }^{3)}$ Thus, we screened the occurrence of somatic mutations in mtDNA molecules in mouse cells by trapping the mutant mtDNAs into mouse rho-0 cells and succeeded in cloning a cybrid cell line carrying both wild-type mtDNA and $\Delta$ mtDNA. ${ }^{15), 16)}$ The deleted region of $\triangle \mathrm{mtDNA}$ is $4,696 \mathrm{bp}$ with a breaking point from nt 7,759-12,454 and includes 6 tRNA genes and 7 structural genes (Fig. 1A). The mouse $\Delta$ mtDNA is similar to mtDNA with a common deletion, which have been shown to be responsible for the pathogenesis of a mitochondrial encephalomyopathy, Kearns-Sayre syndrome.4),17)

The second point for the successful generation of mice carrying mutant mtDNA is to introduce respiration-deficient mitochondria into mouse zygotes (B6 strain) using an electrofusion technique (Fig. 1B). The respiration-deficient cybrids with a predominant amount of $\Delta$ mtDNA were used as mtDNA donors for generation of mice with $\Delta$ mtDNA. Introduction of $\Delta$ mtDNA into mouse zygotes was attained by electrofusion of pronucleus stage embryos with several enucleated cytoplasts of the cybrids. The embryos fused with cytoplasts were cultured in vitro for $24-48 \mathrm{~h}$, and transferred to the oviduct of pseudopregnant females. Since F0 progeny was healthy due to low load of $\Delta$ mtDNA (5.7\%-13.0\% in tails), we selected F0 females with $\triangle$ mtDNA as mothers for breeding and obtained progenies with predominant amounts of $\Delta \mathrm{mtDNA}$, suggesting the occurrence of $\Delta$ mtDNA transmission through female germ lines from F0 mothers to their progeny.

\section{Clinical and pathological features of mito-mice}

The great advantages of mito-mice are that they all share exactly the same nuclear genome background (B6 strain), and their genetic variation is restricted to the proportions of the introduced pathogenic $\Delta$ mtDNA (Fig. 2). Therefore, mitomice can provide direct evidence that mitochondrial respiration defects induced by the accumulation of $\Delta$ mtDNA are sufficient by themselves for expression of the clinical phenotypes observed in patients with mutated mtDNA. ${ }^{14), 18)}$

In various tissues of mito-mice with high load of $\Delta$ mtDNA, approximately more than $75 \%$, mosaic composition of cells with different cytochrome $c$ oxidase (COX, also known as complex IV) activity appeared. Since three of the $13 \mathrm{COX}$ subunits are encoded in mtDNA, it has been well known that the absence of these subunits or/and tRNAs due to mtDNA mutations leads to COX deficiencies 
A

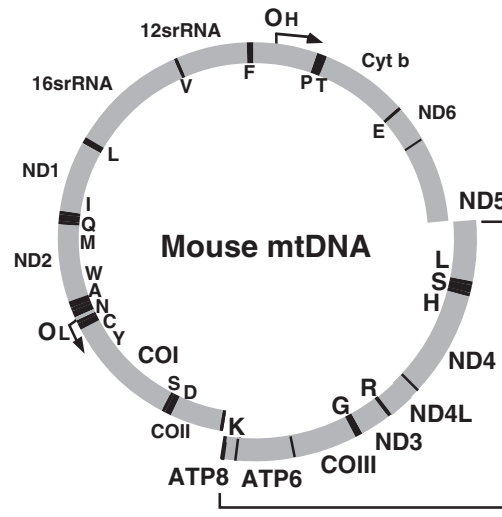

Deleted region of $\triangle \mathrm{mtDNA}$

(4,696bp)

B
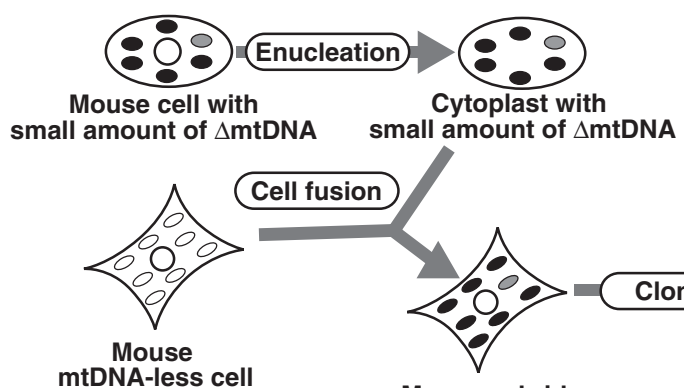

mtDNA-less cell

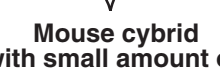

with small amount of

$\triangle$ mtDNA

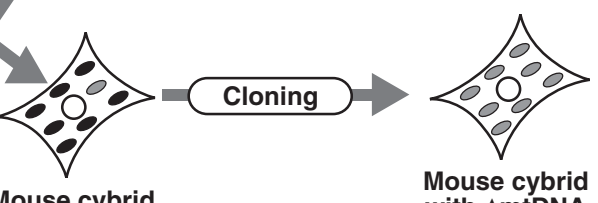

with $A$ mtDNA

Electrofusion

(FnA

Enucleation

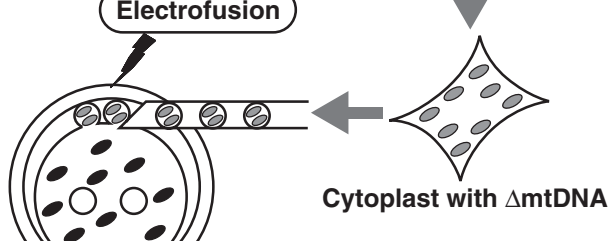

Mouse pronuclear stage embryo

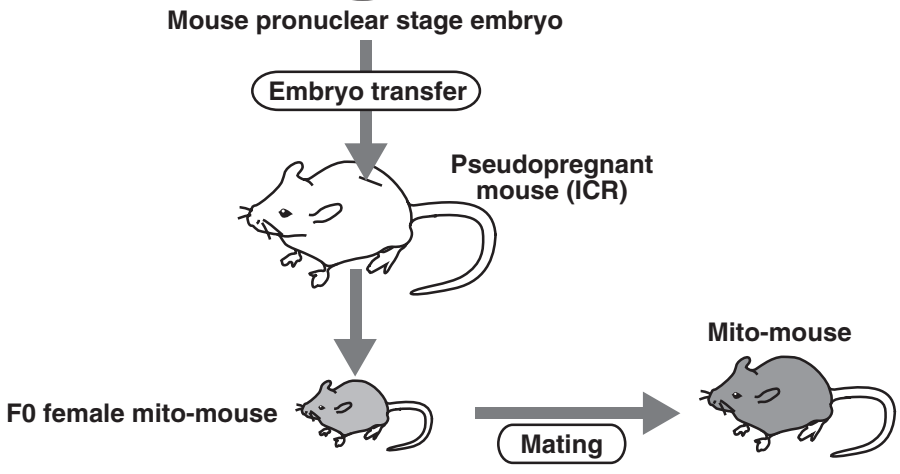

Fig. 1. Genetic characterization of $\triangle \mathrm{mtDNA}$ molecule and generation of the mito-mice.

A, Gene map of mouse mtDNA. The arc indicates the deleted region (4,696 bp) expanded from $t R N A^{L y s}$ to $N D 5$ genes in $\triangle \mathrm{mtDNA} . \mathbf{B}$, Experimental scheme for producing mice carrying wild-type mtDNA and exogenously introduced $\Delta$ mtDNA. In this scheme, mitochondria possessing wild-type mtDNA are shown in a block color, and those with $\Delta$ mtDNA in a gray color. Based on the difference of COX activity, two mitochondrial populations are classified in mouse zygotes after electrofusin. One is COX-positive mitochondria carrying only wild-type mtDNA (black color), which originally exist in mouse zygotes. The other is COX-negative (respiration-deficient) mitochondria carrying predominant amounts of $\Delta$ mtDNA (gray color), which is introduced into mouse zygotes from mouse cybrids. 


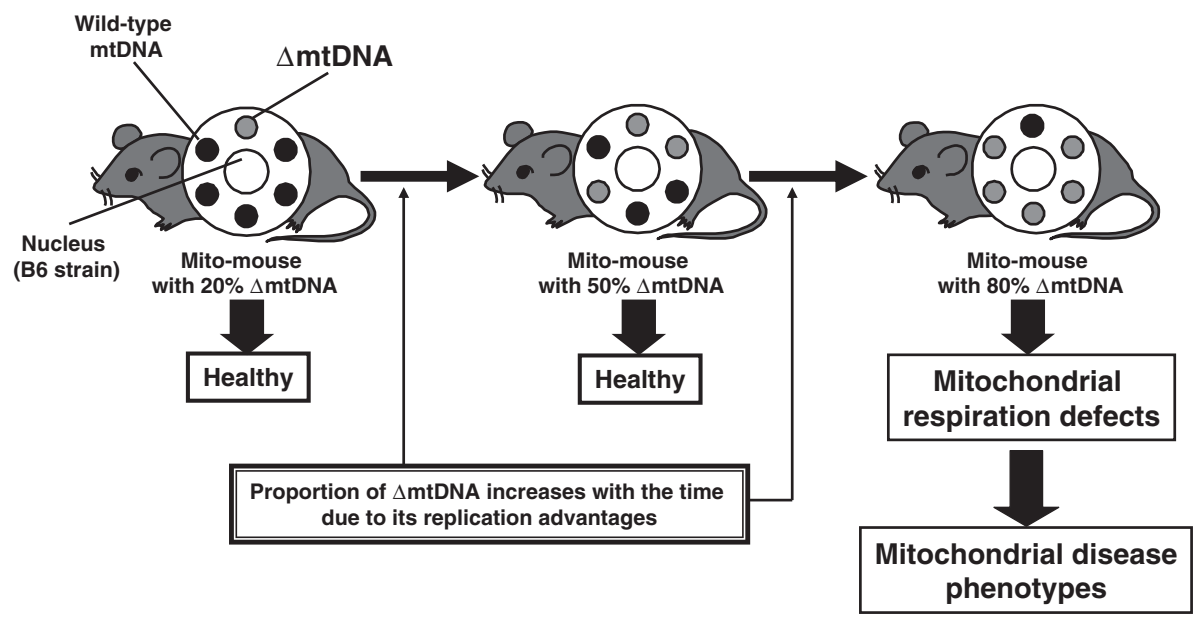

Fig. 2. Biological features as model systems of mito-mice.

Mito-mice share the same nuclear genome background (B6 strain), and their variations are proportions of pathogenic $\Delta$ mtDNA. In these mito-mice, mitochondrial respiration defects and the resultant diseases phenotypes were induced only when $\Delta$ mtDNA accumulated more than $80 \%$ in various tissues. Interestingly, healthy mito-mice carrying low load of $\Delta$ mtDNA would show mitochondrial respiration defects and the resultant diseases phenotypes with aging, because proportion of $\Delta$ mtDNA increases with the time. As a disadvantage in the use of mito-mice, it is mentioned that to obtain a large population with the same $\Delta \mathrm{mtDNA}$ load is difficult.

in cells and tissues. ${ }^{4), 14), 17)}$ In cardiac muscle tissues carrying $88 \% \Delta$ mtDNA, for example, we observed three types of cells, COX-positive, COX-intermediate, and COX-negative cells. ${ }^{18)}$ Quantitative PCR analysis demonstrated that the COX activity in individual cardiac cells were coordinated with the amount of $\triangle$ mtDNA; COX-positive, COX-intermediate, and COX-negative cells carried $76 \%, 83 \%$, and $91 \% \Delta$ mtDNA, respectively ${ }^{19)}$ (see Fig. 3).

Mito-mice with high load of $\Delta$ mtDNA showed also other mitochondrial disease phenotypes due to mitochondrial respiration defects, such as low body weight, lactic acidosis, systemic ischemia, auriculoventricular block with Wenckebach periodicity, hearing loss, renal failures, and male infertility. ${ }^{14), 18), 20)-22)}$ Lactic acidosis is one of the typical clinical phenotypes in patients with mitochondrial respiration defects, and the symptom suggests acceleration of glycolytic pathway for producing ATP without mitochondrial respiration. Early symptoms of the lactic acidosis, low body weight, and sperm abnormalities could be detected in mito-mice carrying approximately more than $75 \%$ $\Delta$ mtDNA. ${ }^{14), 22)}$

$\Delta$ mtDNA load is different among the progeny, and proportion of $\triangle \mathrm{mtDNA}$ in cells and tissues increased with the time ${ }^{14), 23)}$ (Fig. 2). In the case of mito-mice carrying more than $70 \% \Delta$ mtDNA at the birth, they showed mitochondrial respiration defects and disease phenotypes and consequently died on around 6 months after birth. ${ }^{14), 18), 20)-22)}$ On the other hand, mito-mice carrying about 30\%-50\% $\triangle$ mtDNA at the birth were healthy, but they expressed mitochondrial respiration defects and disease phenotypes and died by around 1.5 years after birth when $\triangle$ mtDNA load became $75 \%-$ 85\%. ${ }^{14), 18), 20)-22)}$ Thus, mitochondrial respiration defects and the resultant clinical phenotypes were expressed only when proportion of $\Delta \mathrm{mtDNA}$ exceeded approximately $80 \%$, showing the existence of threshold effects in pathogeneses of mitochondrial diseases (Fig. 3).

Mito-mice carrying a higher amount of $\Delta$ mtDNA did not express diabetic phenotypes, and they showed hypoglycemia caused by enhanced glycolysis. ${ }^{20}$ This result is unexpected and not consistent with the conventional hypothesis of mitochondrial diabetes, which claims that respiration defects caused by mutant mtDNAs in pancreatic beta-cells, skeletal muscle and liver tissues are responsible for their reduced insulin secretion and resistance of insulin and glucose, respectively, leading to expression of diabetic phenotypes. ${ }^{3), 24)}$ We cannot yet explain the exact mechanism or 


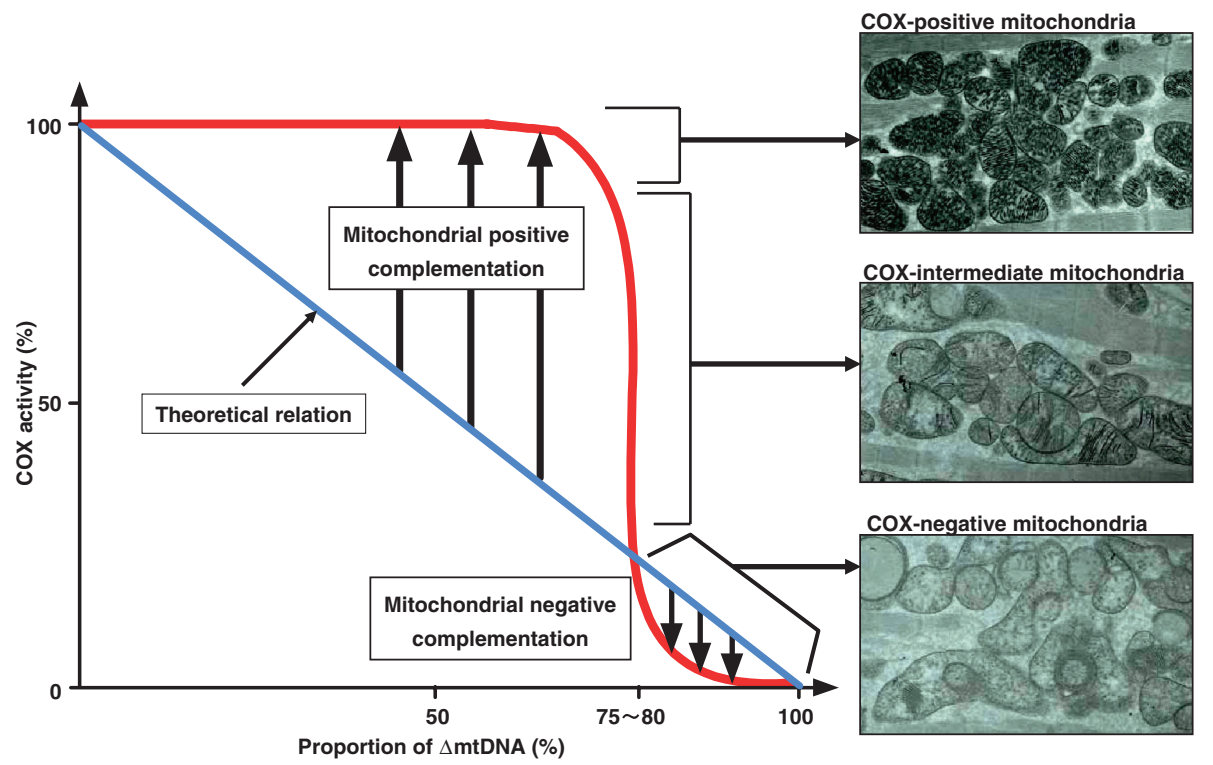

Fig. 3. Regulation mechanisms of pathogeneses in mitochondrial diseases due to mutated mtDNA.

A left graph indicates the relationship between $\triangle$ mtDNA load and COX activity in mito-mice. Theoretically, it is considered that load of 50\% $\triangle$ mtDNA induces 50\% COX activity (blue line in left graph), but there are threshold effects in the relationship (red line in left graph). That is, all mitochondria in single cells carrying less than $75 \% \Delta$ mtDNA show normal mitochondrial respiration activity, while those carrying more than $80 \% \Delta$ mtDNA show respiration defects, due to mitochondrial positive and negative complementation, respectively. In single cells of the mito-mice, uniform composition of COX-positive, COXintermediate, or COX-negative mitochondria is observed (right pictures). Cardiac cells with COX-positive, COX-intermediate, and COX-negative mitochondria possess $76.3 \%, 83.2 \%$, and $91.9 \% \Delta$ mtDNA, respectively.

biological significance of enhanced insulin secretion from pancreatic beta-cells carrying predominant $\Delta$ mtDNA. Possibly the increased level of lactic acid in mito-mice expressing mitochondrial respiration defects could enhance insulin secretion, so that accelerated glucose uptake by respiration-deficient target tissues, such as muscle tissues, protected them from progressive glucose deficiency. Recently, it has been reported that protection of diabetic phenotypes were also observed in mice with mitochondrial respiration defects induced by muscle specific disruption of Tfam gene ${ }^{25}$ ) and apoptosis inducing factor (AIF). ${ }^{26)}$ Thus, the results on mitomice, ${ }^{20)}$ together with mice expressing mitochondrial respiration defects, ${ }^{25), 26)}$ require reassessment of the conventional concept of mitochondrial diabetes, which proposes mtDNA mutations as major pathogenic factors. ${ }^{3), 24)}$

\section{In vivo inter-mitochondrial complementation}

Why could mito-mice with low load of $\Delta$ mtDNA escape mitochondrial respiration defects and the resultant disease phenotypes? We were able to answer this question by using an electron micro- graphic technique that identifies the COX activity at individual mitochondrial levels. ${ }^{18), 19)}$ As shown in Fig. 1B, it can be considered that mouse zygotes that were introduced donor mitochondria carrying $\Delta$ mtDNA possess two mitochondrial populations. One is COX-positive host mitochondrion carrying wild-type mtDNA that originally exists in zygotes. The other is COX-negative donor mitochondrion carrying $\Delta$ mtDNA that is introduced exogenously.

Considering that individual mitochondria in living cells undergo constant migration, fission, and fusion, ${ }^{27)}$ there is a possibility that mitochondria could exchange gene products through their fusion and fission. In this case, all mitochondria in single cells of mito-mice would be COX-positive or COX-negative uniformly (Fig. 4), and exogenous $\triangle$ mtDNA would be detected even in cells with only COX-positive mitochondria. If exchange of gene products did not occur between exogenous donor COX-negative mitochondria carrying $\Delta$ mtDNA and COX-positive mitochondria from recipient zygotes, or even if it occurred, but infrequently and discontinuously, mitochondria in single cells of the mito-mice would continue to show mosaic 

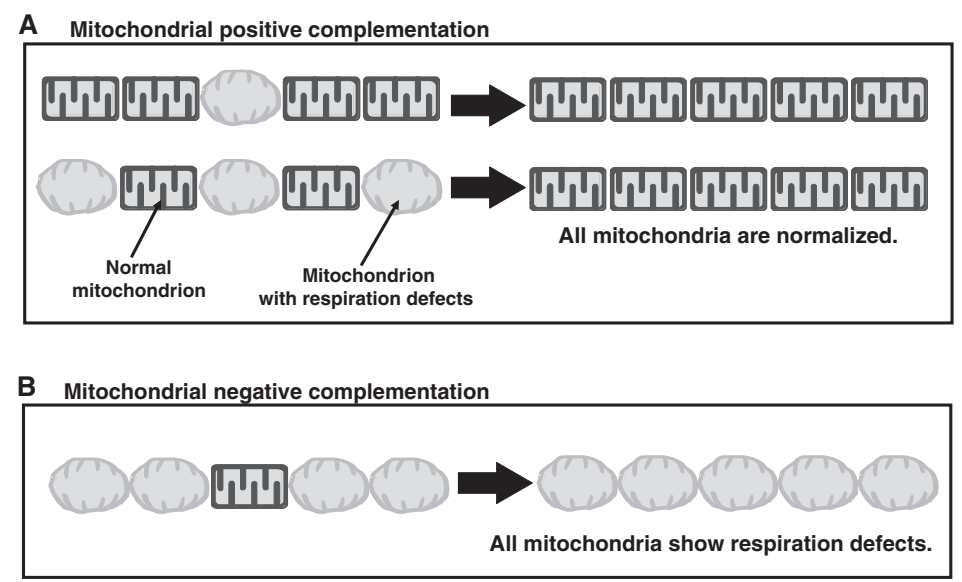

Fig. 4. Schematic presentation of mitochondrial complementation.

Individual mitochondria in single cells can exchange genetic contents via fusion and fission, and they can function as a single dynamic cellular unit in conforming to "one for all, all for one". There are two types of mitochondrial complementation. One is mitochondrial positive complementation (A), which occurs when the number of normal mitochondria is more abounding than that of respiration deficient mitochondria. The other is mitochondrial negative complementation (B), which occurs when the number of respiration-deficient mitochondria is more abounding than that of normal mitochondria. Mitochondrial diseases are just the latter condition. The mitochondrial positive complementation is mitochondria-specific system preventing individual from expression of disease phenotypes by mutated mtDNAs. Black and gray mitochondria indicate normal and respiration-deficient mitochondria, respectively.

distribution of host COX-positive and donor COXnegative mitochondria.

COX electron micrographs of cardiac muscle tissues from mito-mice clearly showed that all mitochondria in single cells with low load of $\Delta \mathrm{mtDNA}$, less than approximately $75 \% \Delta \mathrm{mtDNA}$, were COX-positive (Fig. 3). The appearance of COX-negative mitochondria was limited to cells with more than approximately $85 \% \quad \Delta$ mtDNA (Fig. 3). In cells with $75 \%-85 \% \Delta$ mtDNA load, moreover, COX-intermediate mitochondria were observed (Fig. 3). The appearance of COX-intermediate mitochondria that possess intermediate characters of COX-positive and COX-negative mitochondria indicate the exchange of gene products between COX-positive host mitochondria and COXnegative donor mitochondria. Importantly, it was not observed that the coexistence of COX-positive, COX-intermediate, and COX-negative mitochondria within single cells, irrespective of whether the tissues contained low or high concentrations of $\Delta$ mtDNA (Fig. 3). These observations could be explained by in vivo mitochondrial complementation between COX-negative donor mitochondria carrying $\triangle \mathrm{mtDNA}$ and COX-positive host mitochondria carrying wild-type mtDNA ${ }^{18), 19)}$ (Fig. 4).

Since a threshold level of exogenous $\Delta$ mtDNA required to cause mitochondrial dysfunction in all mitochondria in single cells that would be more than approximately $80 \%$, mitochondrial respiratory function could be maintained quite normally, if wild-type mtDNA exists more than $20 \%$ in single cells. Although $\Delta$ mtDNA had lost 6 tRNA genes, proteins encoded by $\Delta$ mtDNA could be translated with the help of the corresponding tRNAs transcribed from wild-type mtDNA. In this case, all mitochondria in single cells could show normal mitochondrial respiratory function (mitochondrial positive complementation) (see Fig. 4).

On the contrary, in somatic cells where the proportion of wild-type mtDNA is less than 20\%, mitochondrial translation may become limiting due to the lack of sufficient tRNAs transcribed solely from the wild-type mtDNA. Thus, the translation phase may be shifted from complementation to competition of the tRNAs in cells with more than $80 \% \Delta$ mtDNA. In this case, progressive inhibition of overall mitochondrial translation and subsequent reduction of the mitochondrial respiratory function would be induced in all mitochondria of single cells (mitochondrial negative complementation) (see Fig. 4), leading to the onset of the disease phenotypes in various tissues.

The occurrence of frequent and continuous 
interaction throughout mitochondria in living mice ${ }^{18), 19), 28)}$ and human cultured cells ${ }^{29)}$ provided two new concepts on the mitochondrial genetic system in living animals. One was the loss of the individuality of each mitochondrion within single cells. Mammalian cells have been thought to contain hundreds of independent mitochondria, but our observations gave a totally different view that mitochondria function as a single dynamic cellular unit. The other important concept was a novel defense system in mitochondria for avoiding phenotypic expression of respiration defects caused by pathogenic mutant mtDNAs accumulated in somatic tissues. The mitochondrial theory of aging proposed that age-associated accumulatzion of somatic mutations in mtDNAs is responsible for age-associated mitochondrial dysfunction, ${ }^{30)-33)}$ since mtDNA is a target of most carcinogens and mutagens and is continuously exposed to reactive oxygen species produced in mitochondria. In fact, accumulation of various kinds of acquired mutations in mtDNAs with age was reported in human subjects. ${ }^{34)-39)}$ However, this mitochondrial theory of aging is based on above circumstantial evidence, and there have been no reports directly proving that mtDNAs in aged subjects could induce ageassociated mitochondrial defects. ${ }^{40)-43)}$ We found that extensive and continuous interaction occurred between mitochondria in various somatic tissues of mice with $\Delta$ mtDNA, resulting in protection of mice from expressing severe respiration defects.

Based on these views, we would like to propose a novel hypothesis on mitochondrial biogenesis, "interaction theory of mammalian mitochondria": they exchange gene products, and thus lose the individuality and function as a single dynamic cellular unit (Fig. 4). This hypothesis has been confirmed by creation of recombinant mtDNA molecules in vivo. For examination of in vivo mtDNA recombination, mito-mouse possessing $\Delta$ mtDNA of Mus musculus domesticus and wildtype mtDNA of Mus spretus ${ }^{28)}$ were used. To avoid PCR jumping artifacts, PCR products were not used, but whole length mtDNAs purified from mitomouse tissues by EtBr-CsCl centrifugation were used for cloning and sequence analyses. Of the 318 mtDNA molecules, only three molecules possessed specific mutation sites of both $M . m$. domesticus and M. spretus mtDNAs. ${ }^{44)}$ Presence of recombinant mtDNA indicates that the exogenous and endogenous mtDNAs come into close enough proximity to convert their sequences.

\section{New insights for understanding the energy dependency in biological processes using mito-mice}

Mito-mice can be used as model systems for understanding in vivo energy-dependent checkpoints. ${ }^{21), 22)}$ Since most mice with the genetic disruption of nuclear DNA-encoded proteins functional for mitochondrial biogenesis showed embryonic lethal phenotypes, ${ }^{8)-10)}$ it is rather difficult to examine the significance of energy supplied from mitochondrial respiration in in vivo events, such as cell differentiation, maintenance, and regeneration. On the other hand, mito-mouse embryos with low load of $\Delta$ mtDNA can escape from the lethal phenotypes, ${ }^{14), 18)}$ because these embryos do not express mitochondrial respiration defects, due to mitochondrial positive complementation ${ }^{18)}$ (see Fig. 4).

Using mito-mice, we have recently succeeded in showing experimental evidence for mtDNA-based male infertility, and showing that the mitochondrial respiration activity is essential for the mammalian spermatogenesis, especially for progression to the pachytene stages during meiosis (Fig. 5A and $5 \mathrm{~B}){ }^{22)}$ It has been reported that synapsis of homozygous chromosomes in the mammalian spermatogenic meiotic process begins at the zygotene stage through chromosome movement and attachment ${ }^{45), 46)}$ and that several factors for synaptonemal complexes of homozygous chromosomes, such as RAD51, DMC1, and cohesin, contain functional ATP binding domains. ${ }^{47-49)}$ Actually, abnormal and incomplete attachment of homozygous chromosomes occurred only in mito-mice with high load of $\Delta$ mtDNA (Fig. $5 \mathrm{C}$ ). Our in vivo study using mito-mice directly demonstrates that there is an energy-dependent checkpoint in meiotic zygotene stage during mammalian spermatogenesis, and that meiotic arrest due to pathogenic mtDNA-derived mitochondrial respiration defects results in male infertility. On the basis of these findings, we have proposed that some cases of human male infertility with unknown etiology might result from mitochondrial respiratory dysfunction.

Since the $\Delta$ mtDNA load differs in each cell, spermatogenic cells carrying a relatively lower proportion of $\Delta$ mtDNA can complete meiosis and 

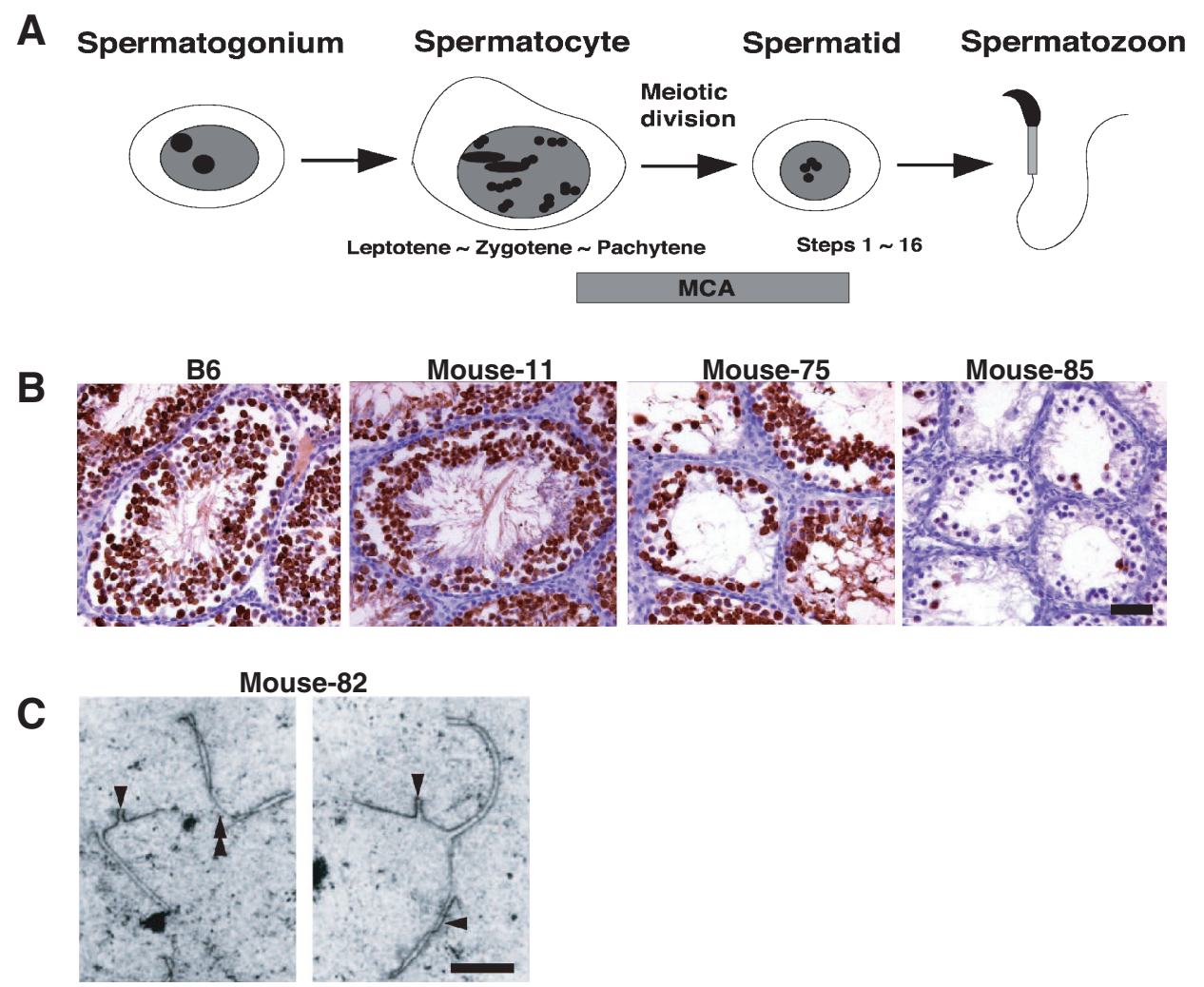

Fig. 5. Pathological observations of mito-mouse testes.

A, Schematic presentation of mammalian spermatogenesis. Mammalian spermatogenesis occurs continuously with individual maturation of sperm and comprises the entire sequence of events by which spermatogonia are transformed into sperm, through meiotic division of spermatocytes. MCA indicates expression of male meiotic metaphase chromosome-associated acidic protein (meichroacidin). B, Distribution of spermatogenic cells in the state of meiotic processes. To visualize spermatocytes and spermatids, testis sections from B6 mouse, Mouse-11, Mouse-75, and Mouse-85 were stained with anti-MCA antiserum, because anti-MCA is specific for the cytoplasm of spermatocytes undergoing meiosis and early round spermatids, but not for cytoplasm of other cells in the testis (see Fig. 5A). Fewer cells stained positive for the anti-MCA decreased in Mouse-75 testis than in Mouse-11 or B6 mouse testes and the number of such cells further decreased in Mouse-85, indicating meiotic abnormalities in spermatogenesis. The Mouse-11, Mouse- 75 , and Mouse- 85 possessed 11\%, 75\%, and $85 \% \Delta$ mtDNA in their tails. Scale bar, $50 \mu \mathrm{m}$. C, Homozygous chromosomes at the zygotene stage in Mouse-82. Abnormal homozygous chromosomes were seen in spermatocyte nuclei at the zygotene stage (see Fig. 5A). Single and double arrowheads indicate the region with self-attachment and the degenerated chromosome, respectively. The homozygous chromosomes in a right picture were avoided to form their synapsis due to the self-attachment. The Mouse- 82 possessed $82 \% \Delta$ mtDNA in the tail. Scale bar, $2 \mu \mathrm{m}$.

transformation into sperms, but the number and motility are reduced. ${ }^{22)}$ In comparison with disease model mice generated by the manipulation of the nuclear genome, mito-mice are not always suitable for the drug screening, because it is difficult to obtain a large population of mito-mice with the same $\triangle$ mtDNA load. Sperm samples from mitomice could be utilized for large-scale screening drugs designed to restore mitochondrial respiratory dysfunction, based on the recovery of decreased sperm motility. Moreover, drugs capable of improving mitochondrial respiratory dysfunction would be useful for treating not only mitochondrial diseases but also male infertility from mitochondrial causes. ${ }^{22)}$

\section{Gene therapy of mito-mice by nuclear transplantation}

Considering the pathogeneses of mitochondrial diseases regulated by mitochondrial complementation, dilution of pathogenic mutated mtDNA population is one of the prenatal therapy strategies for the diseases. Effective procedures to obtain normal progeny from affected mothers would be either 
nuclear transplantation from mito-mouse zygotes to enucleated normal zygotes, or cytoplasmic transplantation of mitochondria from normal to mitomouse zygotes. ${ }^{50), 51)}$ Ooplasmic transfer has been used as an effective technique to restore normal developmental potential of oocytes from patients with embryonic development failure. ${ }^{52), 53)}$ However, the amount of mtDNA copies that could be introduced into mouse zygotes by cytoplasmic transplantation is relatively small.

Nuclear transplantation inevitably co-introduces a small volume of cytoplasm and mitochondria, but the amount of mtDNA co-introduced with nucleus is $6 \%$ of all mtDNA in zygotes. ${ }^{54)}$ Theoretically, nuclear transplantation would result in production of nuclear transplanted-zygotes with $6 \% \Delta$ mtDNA from mito-mouse zygotes, even when the mito-mouse zygotes possess $100 \% \Delta$ mtDNA. Since the maximum proportion of $\Delta \mathrm{mtDNA}$ in mito-mouse zygotes was $78 \%$, nuclear transplantation therapy could hold the proportion of co-transplanted $\Delta$ mtDNA at $5 \%$ or less. Therefore, nuclear transplantation was considered to be more effective than cytoplasmic transplantation for sufficient dilution of $\Delta$ mtDNA in zygotes of mito-mice.

In nuclear transplantation therapy, we first estimated proportions $\Delta$ mtDNA in mito-mouse zygotes using polar bodies as biopsy samples. Then, from 39 zygotes possessing 35\% $\Delta$ mtDNA, karyoplasts were prepared and fused with enucleated normal zygotes. They were transferred to pseudopregnant females, and the resultant 11 mice at the weaned stage possessed $11 \% \Delta$ mtDNA in their tails. On the other hand, when 34 mito-mouse zygotes with $32 \% \Delta$ mtDNA were transferred without nuclear transplantation, obtained 9 mice possessed $66 \% \Delta$ mtDNA. They showed mitochondrial respiration deficiencies in various tissues and died due to renal failure at 218-277 days after birth, while nuclear-transplanted mito-mice showed no abnormalities throughout their lives. ${ }^{54)}$

In human cases, however, there are several biological problems to be resolved before applying gene therapy to zygotes. One is that in human cases maternal transmission of mtDNA with a common deletion is rare. ${ }^{3)}$ When deletion mutant mtDNA is transmitted to oocytes, ${ }^{55}$ the 13 times longer gestation period in humans than in mice would result in accumulation of a sufficient proportion of deletion mutant mtDNA in nuclear-transplanted embryos to be lethal before birth. Therefore, application of nuclear transplantation would be restricted to patients with mitochondrial diseases, only when pathogenic mtDNAs in patients were inherited maternally, and did not possess significant replication advantages over wild-type mtDNA. For example, zygotes from patients with MELAS (mitochondrial myopathy, encephalopathy and lactic acidosis, and stroke-like episodes) and MERRF (myoclonus epilepsy associated with ragged-red fibers) caused by point mutations in mtDNAs, respectively, would be appropriate for applying nuclear transplantation to rescue their progeny. In these cases, a small proportion of wild-type mtDNAs was sufficient to suppress disease phenotypes. ${ }^{56), 57)}$ The other problem is that nuclear transplantation results in zygotes possessing the heteroplasmic state of mtDNA molecules from two mothers. At the present stage, we could not rule out the possibility that the heteroplasmic state itself or the resultant creation of mtDNA recombinants might induce mitochondrial abnormalities.

Recently, it was reported that the UK Human Fertilization and Embryology Authority (HFEA) would allow scientists at the University of Newcastle upon Tyne to transfer the nucleus of a human fertilized egg into an egg donated by a second woman. Although the study using mito-mice unambiguously showed that the nuclear transplantation technique could also be applied to human patients with mitochondrial diseases as a prenatal therapy, we would like to stress that we have to discuss not only biological safety, but also ethical points in the application of this therapy.

\section{Future works}

All genes encoded by human mtDNA are required for mitochondrial respiratory function, so that any pathogenic mutations in the mtDNA could induces mitochondrial respiration defects, leading to various clinical phenotypes associated with mitochondrial diseases. Disease phenotypes, however, are different among the types of the mutation. For example, mutations in $t R N A$ genes are responsible for MELAS, MERRF, and cardiomyopathy, whereas ones in structural genes induce Leigh syndrome and Laber's disease. Furthermore, nuclear genome backgrounds could involve in the difference of disease phenotypes, since most subunits for respiration complexes are encoded in 
nuclear genome. For addressing precise pathogeneses in mitochondrial diseases, we have to generate new mito-mice carrying various mutated mtDNAs responsible for human cases and various nuclear genome backgrounds. Recently, we have succeeded in generating new mito-mouse line carrying mtDNA with a point mutation in COXI gene, and this mouse have showed lactic acidosis and growth retardation. ${ }^{58)}$ In addition, we continue to generate mouse lines with mutated mtDNAs, so we will be able to report several mouse models for mtDNAbased diseases in near future.

\section{References}

1) Clayton, D. A. (1982) Cell 28, 693-705.

2) Clayton, D. A. (1984) Annu. Rev. Biochem. 53, 573-594.

3) Wallace, D. C. (1999) Science 283, 1482-1488.

4) Hayashi, J.-I., Ohta, S., Kikuchi, A., Goto, Y.-i. and Nonaka, I. (1991) Proc. Natl. Acad. Sci. USA 88, 10614-10618.

5) Chomyn, A., Meola, G., Bresolin, N., Lai, S. T., Scarlato, G. and Attardi, G. (1991) Mol. Cell. Biol. 11, 2236-2244.

6) King, M. P., Koga, Y., Davidson, M. and Schon, E. A. (1992) Mol. Cell. Biol. 12, 480-490.

7) Yoneda, M., Miyatake, T. and Attardi, G. (1994) Mol. Cell. Biol. 13, 2699-2712.

8) Graham, B. H., Waymire, K. G., Trounce, I. A., MacGreqor, G. R. and Wallace, D. C. (1997) Nat. Genet. 16, 226-234.

9) Li, Y., Huang, T. T., Carlson, E. J., Melov, S., Ursell, P. C., Olson, J. L., Noble, L. J., Yoshimura, M. P., Berger, C., Chan, P. H., et al. (1995) Nat. Genet. 11, 376-381.

10) Larsson, N. G., Wang, J., Wilhelmsson, H., Oldfors, A., Rustin, P., Lewandoski, M., Barsh, G. S. and Clayton, D. A. (1998) Nat. Genet. 18, 231-236.

11) Wang, J., Wilhelmsson, H., Graff, C., Li, H., Oldfors, A., Rustin, P., Bruning, J. C., Kahn, C. R., Clayton, D. A., Barsh, G. S., et al. (1999) Nat. Genet. 21, 133-137.

12) Silva, J. P., Kohler, M., Graff, C., Oldfors, A., Magnuson, M. A., Berggren, P. O. and Larsson, N. G. (2000) Nat. Genet. 26, 334-336.

13) Sorensen, L., Ekstrand, M., Silva, J. P., Lindqvist, E., Xu, B., Rustin, P., Olson, L. and Larrson, N. G. (2001) J. Neurosci. 21, 8082-8090.

14) Inoue, K., Nakada, K., Ogura, A., Isobe, K., Goto, Y.-i., Nonaka, I. and Hayashi, J.-I. (2000) Nat. Genet. 26, 176-181.

15) Inoue, K., Ito, S., Takai, D., Soejima, A., Shisa, H., LePecq, J. B., Segal-Bendirdjian, E., Kagawa, Y. and Hayashi, J.-I. (1997) J. Biol. Chem. 272, 15510-15515.

16) Inoue, K., Takai, D., Hosaka, H., Ito, S., Shitara, H., Isobe, K., LePecq, J. B., Segal-Bendirdjian,
E. and Hayashi, J.-I. (1997) Biochem. Biophys. Res. Commun. 239, 257-260.

17) Holt, I. J., Harding, A. and Morgan-Hughes, J. A. (1988) Nature 331, 717-719.

18) Nakada, K., Inoue, K., Ono, T., Isobe, K., Ogura, A., Goto, Y.-i., Nonaka, I. and Hayashi, J.-I. (2001) Nat. Med. 7, 924-939.

19) Nakada, K. Inoue, K., Chen, C. S., Nonaka, I., Goto, Y.-i. and Hayashi, J.-I. (2001) Biochem. Biophys. Res. Commun. 288, 901-907.

20) Nakada. K., Sato, A., Sone, H., Kasahara, A., Ikeda, K., Kagawa, Y., Yonekawa, H. and Hayashi, J.-I. (2004) Biochem. Biophys. Res. Commun. 323, 175-184.

21) Inoue, S.-I., Yokota, M., Nakada, K., Miyoshi, H. and Hayashi, J.-I. (2007) FEBS Lett. 581, 19101916.

22) Nakada, K., Sato, A., Yoshida, K., Morita, T., Tanaka, H., Inoue, S.-I., Yonekawa, H. and Hayashi, J.-I. (2006) Proc. Natl. Acad. Sci. USA 103, 15148-15153.

23) Sato, A., Nakada, K., Shitara, H., Kasahara, A., Yonekawa, H. and Hayashi, J.-I. (2007) Genetics 177, 2031-2037.

24) Maechler, C. B. and Wollhein, C. B. (2001) Nature 414, 807-812.

25) Wredenberg, A., Freyer, C., Sandstrom, M. E., Katz, A., Wibom, R., Westerblad, H. and Larsson, N. G. (2006) Biochem. Biophys. Res. Commun. 350, 202-207.

26) Pospisilik, J. A., Knauf, C., Joza, N., Benit, P., Orthofer, M., Cani, P. D., Ebersberger, I., Nakashima, T., Sarao, R., Neely, G. et al. (2007) Cell 131, 476-491.

27) Dimmer, K. S. and Scorrano, L. (2006) Physiology 21, 233-241.

28) Sato, A., Nakada, K., Shitara, H., Yonekawa, H. and Hayashi, J.-I. (2004) Genetics 167, 18551861.

29) Ono, T., Isobe, K., Nakada, K. and Hayashi, J.-I. (2001) Nat. Genet. 28, 272-275.

30) Trounce, I., Byrne, E. and Marzuki, S. (1989) Lancet 1, 637-639.

31) Wallace, D. C. (1992) Science 256, 628-632.

32) Shigenaga, M. K., Hagen, T. M. and Ames, B. N. (1994) Proc. Natl. Acad. Sci. USA 91, 10771-10778.

33) Nagley, P. and Wei, Y.-H. (1998) Trends Genet. 14, 513-517.

34) Linnane, A. W., Marzuki, S., Ozawa, T. and Tanaka, M. (1989) Lancet 1, 642-645.

35) Corral-Debrinski, M., Horton, T., Lott, M. T., Shoffner, J. M., Beal, M. F. and Wallace, D. C. (1992) Nat. Genet. 2, 324-329.

36) Soong, N. W., Hinton, D. R., Cortopassi, G. and Arnheim, N. (1992) Nat. Genet. 2, 318-323.

37) Laderman, K. A., Penny, J. R., Mazzucchelli, F., Bresolin, N., Scarlato, G. and Attardi, G. (1996) J. Biol. Chem. 271, 15891-15897.

38) Liu, V. W., Zhang, C. and Nagley, P. (1998) Nucleic Acids Res. 26, 1268-1275.

39) Michikawa, Y., Mazzucchelli, F., Bresolin, N., 
Scarlato, G. and Attardi, G. (1999) Science 286, 774-779.

40) Hayashi, J.-I., Ohta, S., Kagawa, Y., Kondo, H., Kaneda, H., Yonekawa, H., Takai, D. and Miyabayashi, S. (1994) J. Biol. Chem. 269, $6878-6883$.

41) Isobe, K., Ito, S., Hosaka, H., Iwamura, Y., Kondo, H., Kagawa, Y. and Hayashi, J.-I. (1998) J. Biol. Chem. 273, 4601-4606.

42) Ito, S., Inoue, K., Yanagisawa, N., Kaneko, M. and Hayashi, J.-I. (1998) Biochem. Biophys. Res. Commun. 247, 432-435.

43) Ito, S., Ohta, S., Nishimaki, K., Kagawa, Y., Soma, R., Kuno, S.-Y., Komatsuzaki, Y., Mizusawa, H. and Hayashi, J.-I. (1999) Proc. Natl. Acad. Sci. USA 96, 2099-2103.

44) Sato, A., Nakada, K., Akimoto, M., Ishikawa, K., Shitara, H., Yonekawa, H. and Hayashi, J.-I. (2005) Proc. Natl. Acad. Sci. USA 102, 6057-6062.

45) Barton, N. R. and Goldstein, L. S. B. (1996) Proc. Natl. Acad. Sci. USA 93, 1735-1742.

46) Cobb, J. and Handel, M. A. (1998) Semi. Cell Dev. Biol. 9, 445-450.

47) Morita, T., Yoshimura, Y., Yamamoto, A., Murata, K., Mori, M., Yamamoto, H. and Matsushiro, A. (1993) Proc. Natl. Acad. Sci. USA 90, 6577-6580.

48) Habu, T., Taki, T., West, A., Nishimune, Y. and Morita, T. (1996) Nucleic Acids Res. 24, 470-477.

49) Anderson, D. E., Losada, A., Erickson, H. P. and
Hirano, T. (2002) J. Cell Sci. 156, 419-424.

50) Taylor, R. W. and Turnbull, D. M. (2005) Nat. Rev. Genet. 6, 389-402.

51) Thorburn, D. R. and Dahl, H. H. (2001) Am. J. Med. Genet. 106, 102-114.

52) Cohen, J., Scott, R., Schimmel, T., Levron, J. and Willadsen, S. (1997) Lancet 19, 186-187.

53) Cohen, J., Scott, R., Alikani, R. M., Schimmel, T., Munne, S., Levron, J., Wu, J., Brenner, C., Warner, C. and Willadsen, S. (1998) Mol. Hum. Reprod. 4, 269-280

54) Sato, A., Kono, T., Nakada, K., Ishikawa, K., Inoue, S.-I., Yonekawa, H. and Hayashi, J.-I. (2005) Proc. Natl. Acad. Sci. USA 102, 1676516770 .

55) Marchington, D. R., Macaulay, V., Hartshorne, G. M., Barlow, D. and Poulton, J. (1998) Am. J. Hum. Genet. 63, 769-775.

56) Shoffner, J. M., Lott, M. T., Lezza, A. M., Seibel, P., Ballinger, S. W. and Wallace, D. C. (1990) Cell 61, 931-937.

57) Chomyn, A., Martinuzzi, A., Yoneda, M., Daga, A., Hurko, O., Johns, D., Lai, S. T., Nonaka, I., Angelini, C. and Attardi, G. (1992) Proc. Natl. Acad. Sci. USA 89, 4221-4225.

58) Kasahara, A., Ishikawa, K., Yamaoka, M., Ito, M., Watanabe, N., Akimoto, M., Sato, A., Nakada, K., Endo, H., Suda, Y. et al. (2006) Hum. Mol. Genet. 15, 871-881.

(Received Jan. 31, 2008; accepted Mar. 27, 2008)

\section{Profile}

Kazuto Nakada was born in Tochigi, 1969. He received his Ph.D. from University of Tsukuba in 1999 under the supervision of Prof. Tamio Hirabayashi. After working at Prof. Hirabayashi's laboratory as a Research Fellow at the Japan Society for the Promotion of Science (JSPS), he was promoted to Assistant Professor of University of Tsukuba in 2000, and he started mitochondrial studies at Prof. JunIchi Hayashi's laboratory. For 3 years from 2001, he also worked as a Researcher at Precursory Research for Embryonic Science and Technology (PRESTO), Japan Science and Technology Agency. Since 2004, he is Associate Professor of Cell Biology at University of Tsukuba. He received Young Scientist Award of the Ministry of Education, Culture, Sports, Science and Technology-Japan in 2006 and

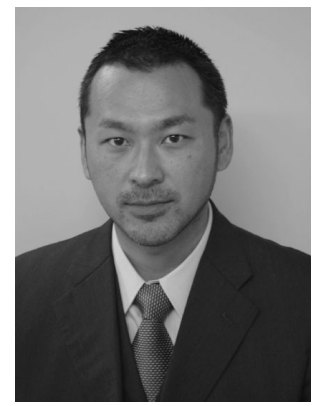
JSPS Prize in 2008. His research interest focuses on biogenesis of mammalian mitochondria carrying mutated mitochondrial genome. 